\title{
Comparative Study of Principal Component Analysis and Independent Component Analysis
}

\author{
Sushma Niket Borade \\ Research Scholar \\ Dept. of Computer Science and I.T. \\ Dr. Babasaheb Ambedkar Marathwada University, \\ Aurangabad - 431004, India
}

\author{
Ratnadeep R. Deshmukh, Ph. D \\ Professor \\ Dept. of Computer Science and I.T. \\ Dr. Babasaheb Ambedkar Marathwada University, \\ Aurangabad - 431004, India
}

\begin{abstract}
Face recognition is emerging as an active research area with numerous commercial and law enforcement applications. This paper presents comparative analysis of two most popular subspace projection techniques for face recognition. It compares Principal Component Analysis (PCA) and Independent Component Analysis (ICA), as implemented by the InfoMax algorithm. ORL face database is used for training and testing of the system. The results show that for the task of face recognition, ICA outperforms PCA in terms of recognition rate and subspace dimensionality.
\end{abstract}

\section{General Terms}

Image processing, Pattern Recognition, Face Recognition.

\section{Keywords}

Face recognition, PCA, ICA, subspace analysis methods.

\section{INTRODUCTION}

A biometric system provides automatic identification for an individual based on a unique feature or characteristics possessed by the individual. Biometric systems have been developed based on eye, iris, retina, fingerprints, finger geometry, voice, hand geometry, palm, signature and the one presented in this paper, the human face. Among these systems, fingerprint analysis and retinal or iris scans are very reliable. But they rely on the cooperation of the participant, whereas face recognition system is effective because it does not require participant's cooperation or knowledge. Face recognition is emerging as an active research area in the fields of image processing, pattern recognition, computer vision and neural network. Its applications range from static matching of controlled format photographs such as passports, credit cards, photo IDs, drivers licenses, and mug shots to real time matching of surveillance video images. Face recognition problem can be defined as the identification of one or more persons using stored database of face images. Zhao et al. has given detailed literature survey on face recognition techniques [1]. Face recognition methods [2] are categorized as: 1) Appearance-based or template matching methods: They compare the intensity values of the whole face or specific regions of face with the intensity values of the ones in the database. 2) Feature-based or structural matching methods: They extract the local features (brows, eyes, nose, mouth, cheeks etc.) and form the feature vectors using distances and angles between them.

Among various approaches to face recognition, appearancebased subspace methods give the most promising results. In these subspace projection techniques, probe image is projected on lower dimensional subspace and then recognition is done by finding its distance from gallery images. In this paper, two most popular subspace projection techniques for face recognition are presented. They are: Principal Component Analysis (PCA) and Independent Component Analysis (ICA). PCA finds basis vectors such that when samples are projected on them, they retain most of the original information [3, 4]. ICA decorrelates both second and high order statistics of data and finds statistically independent basis vectors [5]. Basically PCA considers the $2^{\text {nd }}$ order moments only and it uncorrelates data, while ICA also uncorrelates higher order statistics and identifies the independent sources from their mixtures.

This paper provides a comparative analysis of these two projection methods in equal working environment. Comparison is done using grayscale images of persons from ORL face database, which is a publicly available database. For fair comparison, same preprocessed images are used as input into these algorithms. Distance metrics used are Euclidean and cosine.

Why to perform comparative analysis of PCA and ICA? It is interesting to note that the literature on the subject is contradictory. For example, Liu and Wechsler [6], Barlett et al. [5,7], and Yuen and Lai [8] claimed that ICA outperforms PCA for face recognition. However, other research groups reported different findings on this subject. Baek et al. [9] claimed that PCA outperforms ICA. Moghaddam [10] and Jin [11] reported that there is no significant difference between the performances of these two methods. Socolinsky and Salinger recently showed that ICA outperforms PCA on visible light images, but PCA is better than ICA on infrared images [12]. Draper et al. [13] tried to relate previous comparisons to each other and to their work by testing PCA and ICA on FERET database. They showed that the performance of PCA and ICA depends on the task statement, the ICA architecture and the distance metric.

The rest of this paper is organized as follows: Section 2 describes the algorithms to be compared, Section 3 reports the details of methodology, Section 4 presents performance comparison of the described methods and Section 5 concludes the paper.

\section{ALGORITHMS}

\subsection{Principal Component Analysis}

A two-dimension face image with size $N$ by $N$ may be viewed as one dimensional vector of dimension $N^{2}$ or a point in $N^{2}$ dimensional space, called image space, where each coordinate of space corresponds to a pixel of the image. Space derived this way is substantial and recognition in it is computationally inefficient. Actually the intrinsic dimensionality of face space is much smaller due to the similar topology of the face images. Principal Component Analysis is used to reduce the dimensionality of data while retaining variation as much as possible in the original dataset. Sirovich and Kirby [14, 15] 
applied PCA to efficiently represent faces while Turk and Pentland used PCA for representing and recognizing faces. PCA finds a set of orthogonal basis vectors which best reconstructs the face images with smallest mean square error for given subspace. These orthogonal basis vectors are the eigenvectors of the covariance matrix of the face images. Due to the face like appearance of eigenvectors, they are also called as eigenfaces.

In this paper, face recognition using PCA is implemented as proposed by Turk and Pentland $[3,4]$. Let the training set of $M$ face images be $\Gamma_{l}, \Gamma_{2}, \ldots, \Gamma_{M}$ and average face of the set be $\Psi$. Each face differs from the average face by the vector $\Phi_{i}=\Gamma_{i}-\Psi$. Covariance matrix $C$ is found as:

$$
C=A A^{T}
$$

where matrix $A=\left[\Phi_{1} \Phi_{2} \ldots \Phi_{M}\right]$. The size of covariance matrix $C$ is $N^{2}$ by $N^{2}$, and its intractable task for typical image sizes to find its $N^{2}$ eigenvectors and eigenvalues. Therefore Turk and Pentland proposed computationally feasible method to find these eigenvectors. The eigenvectors $v_{i}$ of $A^{T} A$ are considered such that

$$
A^{T} A v_{i}=\mu_{i} v_{i}
$$

Premultiplying both sides by $A$

$$
A A^{T} A v_{i}=\mu_{i} A v_{i}
$$

where $A v_{i}$ are the eigenvectors and $\mu_{i}$ are the eigenvalues of $C=A A^{T}$. Now construct matrix $L=A^{T} A$ which is of size $M$ by $M$, and find its $M$ eigenvectors, $v_{i}$. The eigenvectors of matrix $L$ are used to find most significant $M$ eigenvectors (i.e. eigenfaces) of covariance matrix $C$ as:

$$
u_{l}=\sum_{k=1}^{M} v_{l k} \Phi_{k}, l=1, \ldots, M
$$

This reduces calculations from number of pixels in image $\left(N^{2}\right)$ to number of images $(M)$ in training set. Practically, smaller number of eigenfaces $\left(M^{\prime}\right)$ corresponding to largest eigenvalues are sufficient for face recognition. Eigenvectors corresponding to larger eigenvalues captures more of data variance. The eigenfaces form $M$ dimensional subspace of the original $N^{2}$ image space. Each image in training set is projected on this face space using equation,

$$
w_{k}=u_{k}^{T}(\Gamma-\Psi), \text { for } k=1, \ldots, M^{\prime}
$$

and its projection vector $\Omega^{T}=\left[w_{1}, w_{2}, \ldots w_{M^{\prime}}\right]$ is stored in database. For recognition, the given test image is normalized, mean subtracted and projected on the same subspace. Then its projection vector is compared to the stored projections of training images. Using similarity measure (Euclidean), test image is assigned the identity of closest training image.

\subsection{Independent Component Analysis}

While PCA uncorrelates data using second-order statistics only, ICA minimizes both second-order and high order dependencies of input. ICA is one powerful solution to blind source separation problem where task is to separate original source signals from their mixtures.

\subsubsection{Motivation}

Suppose two people are speaking simultaneously in a room having two microphones. The microphones give two recorded signals, $\mathrm{x}_{1}(t)$ and $\mathrm{x}_{2}(t)$, where $\mathrm{x}_{1}$ and $\mathrm{x}_{2}$ are amplitudes and $t$ is time index. Each recorded signal is a weighted sum of the speech signals, $\mathrm{s}_{1}(t)$ and $\mathrm{s}_{2}(t)$, emitted by the two speakers. This can be expressed as linear equations:

$$
\begin{aligned}
& x_{1}(t)=a_{11} s_{1}+a_{12} s_{2} \\
& x_{2}(t)=a_{21} s_{1}+a_{22} s_{2}
\end{aligned}
$$

where parameter $a_{i j}$ depends on the distances between microphones and the speakers. Here only recorded signals $x_{I}(t)$ and $x_{2}(t)$ are known. The goal is to estimate the two original speech signals $s_{l}(t)$ and $s_{2}(t)$. This is called the cocktail-party problem [16]. Here ICA can be used to estimate the parameters $a_{i j}$. Once $a_{i j}$ are known, the two original source signals $s_{l}(t)$ and $s_{2}(t)$, can be easily separated from their mixtures $x_{l}(t)$ and $x_{2}(t)$.

\subsubsection{ICA Basic Model}

In general, for $n$ mixtures $x_{1} \ldots x_{n}$ of $n$ independent sources, linear equation is:

$$
x_{j}=a_{j 1} s_{1}+a_{j 2} s_{2}+\cdots+a_{j n} s_{n}, \text { for all } j .
$$

Here it is assumed that all mixtures and source signals are random variables instead of time signals. Using matrix notation, this mixing model is written as

$$
X=A S \text {, }
$$

where $A$ is matrix with elements $a_{i j}$. This is called ICA model. Here mixing matrix $A$ is unknown. Only known variable is $X$ and goal is to estimate both $A$ and $S$ using it. It is assumed that source signals $s_{i}$ are statistically independent of each other i.e. value of one does not depend on value of other and unknown mixing matrix $A$ is square and invertible. Once matrix $A$ is obtained, its inverse, say $W$, can be estimated and independent sources can be found by:

$$
S=W X .
$$

ICA can be used for performing blind source separation. A "source" means an original independent signal. "Blind" means very little is known about mixing matrix.

\subsubsection{Preprocessing for ICA}

ICA is generalization of PCA. PCA decorrelates the data so that covariance is zero. This removes first and second order statistics of data. Complexity of problem is reduced by performing dimensionality reduction using PCA and whitening. Whitening is stronger constraint which makes covariance matrix of data equal to identity matrix. For whitening, $X$ is passed through whitening matrix $W_{z}$ which is twice the inverse square root of the covariance matrix as:

$$
W_{z}=2 *(\operatorname{Cov}(X))^{-(1 / 2)}
$$

After whitening, the covariance matrix of $X$ equals the identity matrix [5]. This ensures that mixed signals have unit variance and are uncorrelated. The uncorrelation removes the secondorder dependencies between the observed signals.

\subsubsection{InfoMax Algorithm}

For face recognition various ICA algorithms can be used like InfoMax, FastICA and JADE. Bell and Sejnowski proposed InfoMax algorithm. It is derived from the principle of optimal information transfer in neurons with sigmoidal transfer functions $[17,18]$.

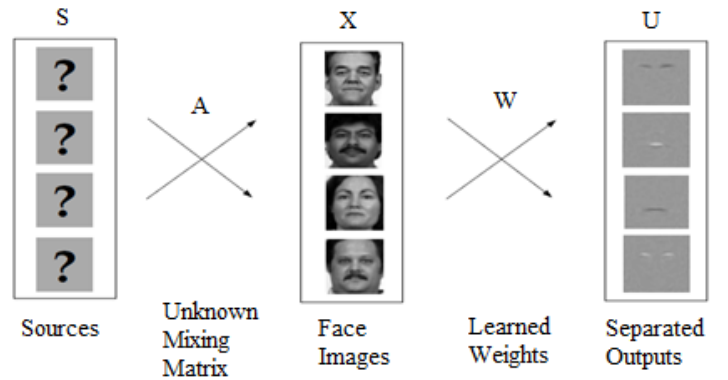

Figure 1: ICA (InfoMax algorithm) - Image synthesis model for architecture I

Let $X$ be vector representing inputs i.e. all images of training set are loaded in matrix $X$ as shown in Figure 1. Each face is made up of linear combination of independent source (i.e. 
basis) images from $S$. Let $W$ be $n$ by $n$ invertible weight matrix. As seen earlier from eq. 10,

$$
U=W X .
$$

Goal is to find matrix $W$ such that $I\left(u_{i}, u_{j}\right)=0$ i.e. mutual information between rows of $U$ is zero.
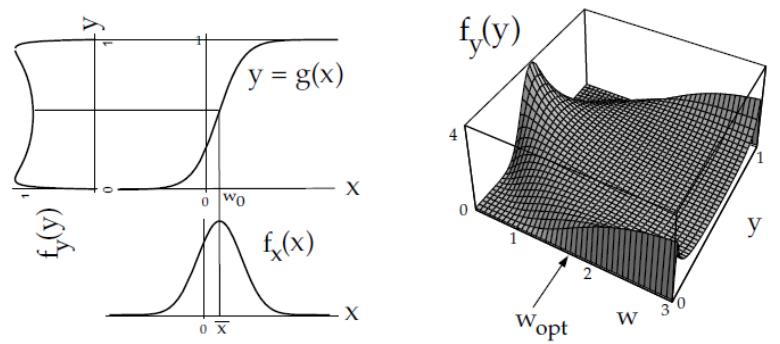

Figure 2: Optimal information flow in sigmoidal neurons. Right: $\mathrm{f}_{\mathrm{y}}(\mathrm{y})$ is plotted for different values of the weight, $w$. The optimal weight, $w_{\text {opt }}$, transmits the most information

If each $u_{i}$ is passed through sigmoidal transfer (i.e. logistic) function as (see Figure 2):

$$
y_{i}=f_{i}(u)=1 /\left(1+e^{-u}\right),
$$

then output $y_{i}$ has maximum entropy. Joint entropy $H\left(y_{i}, y_{j}\right)$ is:

$$
H\left(y_{i}, y_{j}\right)=H\left(y_{i}\right)+H\left(y_{j}\right)-I\left(y_{i}, y_{j}\right) .
$$

Joint entropy is maximum when $I\left(y_{i}, y_{j}\right)=0$. Joint entropy can be maximized by performing gradient ascent on it w.r.t. the weight matrix $W$. The gradient update rule for the $W$ is:

$$
\Delta W=\left(I+Y^{T}\right) W \text {, where } I \text { is identity matrix. }
$$

Now find $W$ for which joint entropy is maximum. When there are multiple inputs and outputs, maximizing the joint entropy of the output $Y$ encourages the individual outputs to move toward statistical independence [5]. Once $W$ is known, $U$ can be found as seen previously:

$$
U=W X
$$

$U$ gives independent basis vectors.

For face recognition, two architectures of ICA are proposed by Barlette et al. [5, 7] as: Architecture I - statistically independent basis images (see Figure 1) and Architecture II factorial code representation. Architecture I treats the images as random variables and the pixels as outcomes and Architecture II treats the pixels as random variables and the images as outcomes.

In this paper, ICA is implemented using InfoMax algorithm which is proposed by Bell and Sejnowski. Architecture I finds a set of independent basis images. All training images are stored in rows of matrix $X$ and $U=W X$. ICA algorithm finds a weight matrix $W$ such that the rows of $U$ are as statistically independent as possible. As seen earlier (Figure 1), rows of $U$ will have independent basis images which will represent faces. The number of Independent Components found by the ICA algorithm corresponds to the dimensionality of the input $(n)$. This is intractable task. Therefore first dimensionality reduction is done using PCA and most significant $m$ eigenvectors of training set are found. Then Independent Component Analysis is performed on these most significant $m$ eigenvectors (instead of performing on all $n$ training images). Here $m<\mathrm{n}$. This produces $m$ independent source (i.e. basis) images in the rows of $U$. Let $P_{m}$ be matrix containing first $m$ eigenvectors in its columns. PC representation of set of images in $X$ based on $P_{m}$ is:

$$
R_{m}=X P_{m}
$$

ICA algorithm produces matrix:

$$
W_{I}=W W_{z},
$$

where $W_{z}$ is a sphering matrix as defined in eq. 11 . The IC representation of face images is given by rows of the matrix:

$$
B=R_{m} W_{I}^{-1} \text {. }
$$

These $b$ 's are some scalar values. Basis vectors are in rows of $U$. Each image in training set $X$ can be represented as weighted linear combination of all independent basis images, $u$, as shown over here.

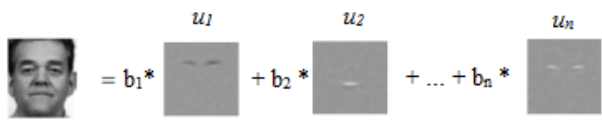

ICA representation $=\left(b_{1}, b_{2}, \ldots, b_{n}\right)$

Figure 3: InfoMax algorithm (architecture-I): The independent basis image representation

\subsubsection{Recognition}

For recognition, ICA representation of test image i.e. $b_{\text {test }}$ is compared with ICA representations of all images in training database using cosine similarity measure as:

$$
c=\frac{b_{\text {test }} \cdot b_{\text {train }}}{\left\|b_{\text {test }}\right\|\left\|b_{\text {train }}\right\|} \text {. }
$$

Test image is then assigned identity of closest training image.

\section{METHODOLOGY}

\subsection{Data}

Standard ORL face database [19] is used for recognition tests. It contains a set of face images taken at the AT \& T Laboratories. It has grayscale images of 40 different persons with 10 images per person. The images are taken by varying the lighting, facial expressions (open / closed eyes, smiling / not smiling) and facial details (glasses / no glasses). All the images are in an upright, frontal position (with tolerance for some side movement). The size of each image is $112 \times 92$ pixels, with 256 grey levels per pixel. Some ORL face database images are shown in Figure 4.

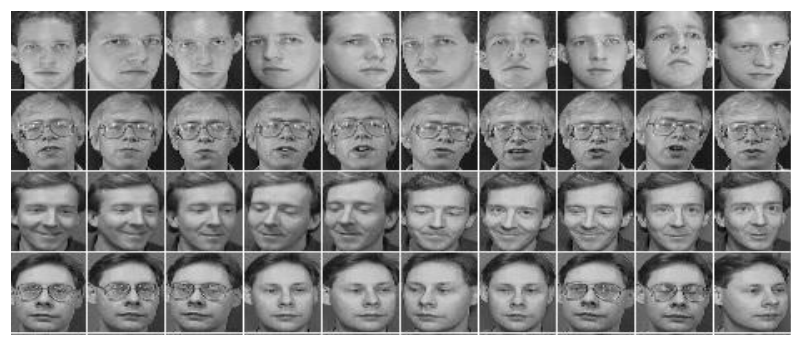

Figure 4: Face samples from the ORL face database

\subsection{Preprocessing and training}

All algorithms and all image preprocessing steps are implemented in MATLAB ${ }^{\circledR}$. Before the recognition process, each image was resized to $50 \mathrm{x} 42$ pixels. System was trained using subset of 150 images from 30 different persons. Five images of each person, with some variation in expression and in the lighting, were randomly selected. Test data set was 
formed by considering one image of each person (from remaining five). It also had five images of unknown persons. Thus, test data set had total 35 images. After all the subspaces had been derived, all images from data sets were projected onto each subspace and recognition using nearest neighbor classification with various distance measures (Euclidean and Cosine) was conducted.

\section{RESULTS}

Results of the experiment can be seen in Table 1 and Figure 5 .

Table 1. Recognition rates corresponding to dimensionality of final subspace for PCA and ICA

\begin{tabular}{|c|c|c|c|}
\hline $\begin{array}{c}\text { Dimensions } \\
\text { of final } \\
\text { subspace }\end{array}$ & $\begin{array}{c}\% \text { of sum of } \\
\text { all } \\
\text { eigenvalues }\end{array}$ & $\begin{array}{c}\text { PCA } \\
\text { (Recognition } \\
\text { rate in \%) }\end{array}$ & $\begin{array}{c}\text { ICA } \\
\text { (Recognition } \\
\text { rate in \%) }\end{array}$ \\
\hline 1 & 20.33 & 14.29 & 0 \\
\hline 2 & 33.60 & 57.14 & 40 \\
\hline 3 & 42.78 & 65.71 & 60 \\
\hline 4 & 49.84 & 77.14 & 71.43 \\
\hline 5 & 56.24 & 71.43 & 65.71 \\
\hline 6 & 60.34 & 80 & 77.14 \\
\hline 7 & 63.79 & 82.86 & 82.86 \\
\hline 8 & 66.55 & 80 & 82.86 \\
\hline 9 & 69.22 & 82.86 & 91.43 \\
\hline 10 & 71.45 & 82.86 & 94.29 \\
\hline 11 & 73.30 & 85.71 & 94.29 \\
\hline 12 & 74.98 & 85.71 & 94.29 \\
\hline 13 & 76.98 & 85.71 & 94.29 \\
\hline 14 & 77.77 & 85.71 & 94.29 \\
\hline 15 & 78.99 & 85.71 & 94.29 \\
\hline 16 & 80.19 & 85.71 & 94.29 \\
\hline 17 & 81.25 & 85.71 & 94.29 \\
\hline 18 & 82.15 & 85.71 & 97.14 \\
\hline 19 & 83.02 & 85.71 & 97.14 \\
\hline 20 & 83.85 & 85.71 & 94.29 \\
\hline 21 & 84.60 & 85.71 & 94.29 \\
\hline 22 & 85.32 & 85.71 & 94.29 \\
\hline 24 & 86.59 & 85.71 & 94.29 \\
\hline 25 & 87.19 & 85.71 & 94.29 \\
\hline 26 & 87.73 & 85.71 & 94.29 \\
\hline 27 & 88.23 & 85.71 & 94.29 \\
\hline 28 & 88.73 & 85.71 & 94.29 \\
\hline 29 & 89.16 & 85.71 & 94.29 \\
\hline 30 & 89.56 & 85.71 & 97.14 \\
\hline 31 & 89.94 & 85.71 & 97.14 \\
\hline 32 & 90.31 & 85.71 & 97.14 \\
\hline 33 & 90.64 & 85.71 & 97.14 \\
\hline 34 & 90.96 & 85.71 & 97.14 \\
\hline 37 & 91.82 & 85.71 & 97.14 \\
\hline 38 & 92.09 & 85.71 & 97.14 \\
\hline 39 & 92.34 & 85.71 & 97.14 \\
\hline 40 & 92.58 & 85.71 & 97.14 \\
\hline 41 & 92.80 & 85.71 & 97.14 \\
\hline 42 & 93.02 & 85.71 & 97.14 \\
\hline 44 & 93.44 & 85.71 & 97.14 \\
\hline 45 & 93.64 & 85.71 & 100 \\
\hline 52 & 94.91 & 85.71 & 100 \\
\hline 59 & 95.90 & 85.71 & 100 \\
\hline 68 & 96.93 & 85.71 & 100 \\
\hline 80 & 97.97 & 85.71 & 100 \\
\hline 90 & 98.63 & 85.71 & 100 \\
\hline 96 & 98.97 & 85.71 & 100 \\
\hline 105 & 99.37 & 85.71 & 100 \\
\hline
\end{tabular}

\begin{tabular}{|c|c|c|c|}
\hline 110 & 99.56 & 85.71 & 100 \\
\hline 120 & 99.85 & 85.71 & 100 \\
\hline 125 & 99.96 & 85.71 & 100 \\
\hline 127 & 99.98 & 85.71 & 100 \\
\hline 128 & 100 & 85.71 & 100 \\
\hline 149 & 100 & 85.71 & 100 \\
\hline
\end{tabular}

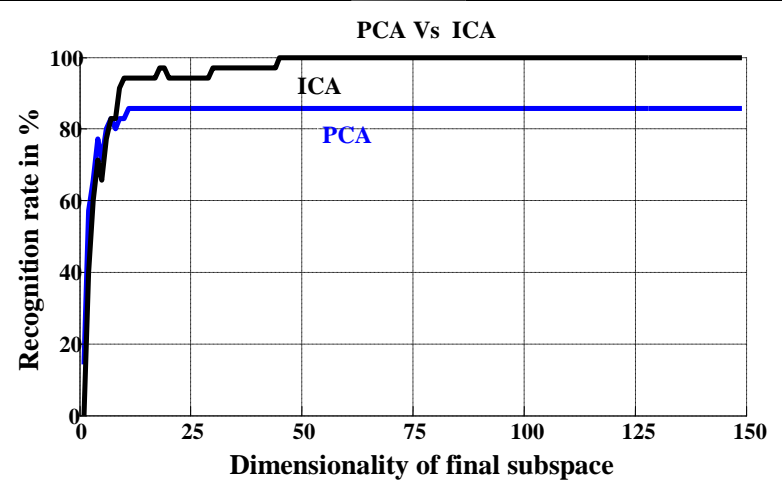

Figure 5: Recognition rates vs. subspace dimensions for PCA and ICA

Recognition rates were measured for subspace dimensionalities starting at 1 and increasing by 1 dimension up to a total of 149. For each approach, it was observed that rate of recognition increased as the dimensionality of final subspace (i.e. number of eigenvectors used) was increased.

As training set consisted of $5 \times 30=150$ images $(M=150)$, PCA derived, in accordance with theory, $M-1=149$ meaningful eigenvectors [3]. For PCA, maximum recognition rate achieved was $85.71 \%$ (using minimum 11 eigenfaces). It was calculated that $73.30 \%$ of original information (energy) was retained in those 11 eigenvectors. Even if the number of eigenfaces in the final subspace is increased, the recognition rate did not increase.

PCA is used as a preprocessing dimensionality reduction step for face recognition using ICA. This PCA subspace was used as input to ICA. $100 \%$ recognition rate was achieved using only 45 eigenvectors. It was calculated that $93.64 \%$ of original information (energy) was retained in subspace dimensionality of 45 . Whereas for face recognition using PCA, maximum recognition rate achieved was $85.71 \%$ (even if all 149 meaningful eigenvectors were considered).

\section{CONCLUSIONS}

This paper presented comparative study of two most popular appearance-based face recognition methods (PCA and ICA) in equal working environments. Following conclusions can be drawn from this research work: (1) Face recognition rate increases as the dimensionality of final subspace increases. (2) ICA greatly improves the performance of face recognition. It outperforms PCA in terms of recognition rate and subspace dimensionality. (3) This is because of the following advantage of using ICA for facial identity: much of the important information is contained in the high-order statistics of the images. In PCA only the second-order statistics are decorrelated. Whereas ICA considers high-order statistical dependencies such as the relationships among three or more pixels and decorrelates both second and high order moments. 


\section{REFERENCES}

[1] Zhao, W., Chellappa, R., and Phillips, A. 2003. Face recognition in still and video images: a literature survey, ACM Comput. Surv. 35 (Dec. 2003), 399-458.

[2] Brunelli, R. and Poggio, T. 1993. Face recognition: features versus templates. IEEE Trans. Pattern Anal. Machine Intell. 15 (Oct. 1993), 1042-1052.

[3] Turk, M. and Pentland, A. 1991. Eigenfaces for recognition. J. Cogn. Neurosci. 3 (1991), 71-86.

[4] Turk, M. and Pentland, A. 1991. Face recognition using eigenfaces. In Proceedings of the IEEE Conference on Computer Vision and Pattern Recognition.

[5] Bartlett, M. S., Movellan, J. R., and Sejnowski, T. J. 2002. Face Recognition by Independent Component Analysis. IEEE Trans. Neural Networks. 13 (Nov. 2002), $1450-1464$

[6] Liu, C. and Wechsler, H. 1999. Comparative assessment of Independent Component Analysis (ICA) for face recognition. Int. Conference on Audio and Video Based Biometric Person Authentication, Washington D.C. USA.

[7] Barlett, M. S., Lades, H. M., and Sejnowski, T. J. 1998. Independent component representations for face recognition. In Proceedings of the SPIE Symposium on Electronic Imaging: Science and Technology Conference on Human Vision and Electronic Imaging III, San Jose, California.

[8] Yuen, P. C. and Lai, J. H. 2000. Independent Component Analysis of face images. IEEE Workshop on Biologically Motivated Computer Vision, Seoul.

[9] Baek, K., Draper, B. A., Beveridge, J. R., and She, K. 2002. PCA vs ICA: A comparison on the FERET data set. Joint Conference on Information Sciences, Durham, NC.

[10] Moghaddam, B. 2002. Principal manifolds and probabilistic subspaces for visual recognition. IEEE
Trans. Pattern Anal. Machine Intell. 24 (June 2002). 780 788.

[11] Jin, Z. and Davoine, F. 2004. Orthogonal ICA representation of images. In Proceedings of the 8th Int. Conference on Control, Automation, Robotics and Vision.

[12] Socolinsky, D. and Selinger, A. 2002. A comparative analysis of face recognition performance with visible and thermal infrared imagery. In Proceedings of the Int. Conference on Pattern Recognition, Quebec City.

[13] Draper, B. A., Baek, K., Bartlett, M. S., and Beveridge, J. R. 2003. Recognizing faces with PCA and ICA. J. Computer vision and image understanding, Special issue on Face Recognition. 91 (July 2003), 115-137.

[14] Sirovich, L. and Kirby, M. 1987. Low-dimensional procedure for the characterization of human faces. J. Optical Society of America. 4 (Mar. 1987), 519-524.

[15] Kirby, M. and Sirovich, L. 1990. Application of the Karhunen-Loeve procedure for the characterization of human faces. IEEE Trans. Pattern Anal. Machine Intell. 12 (Jan. 1990), 103-108.

[16] Hyvarinen, A. and Oja, E. 2000. Independent Component Analysis: algorithms and applications. J. Neural Networks. 13 (June 2000), 411-430.

[17] Bell, A. J. and Sejnowski, T. J. 1995. Fast blind separation based on information theory. In Proceedings of the Int. Symposium on Non-linear Theory and Applications, Las Vegas.

[18] Bell, A. and Sejnowski, T. 1995. An informationmaximization approach to blind separation and blind deconvolution. J. Neural Computation. 7 (Nov. 1995), 1004-1034.

[19] http://www.cl.cam.ac.uk/research/dtg/attarchive/facedata base.html. 